tions of the source at other wavelengths are needed to determine the spectral index and physical parameters of the source.

The entire observatory staff contributed to the observations and reduction of the data. This research was supported by the Office of Naval Research under contract Nonr 1834(22).

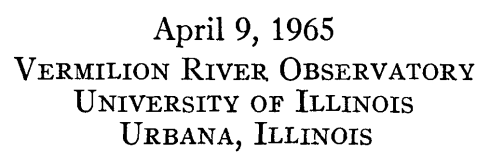

J. R. DickeL

J. P. MCGUIRE

K. S. YANG

\title{
REFERENCES
}

Bergh, S. van den. 1960, $Z$ f. $A p, \mathbf{5 1}, 15$

Hogg, D. E. 1962, A.J., 67, 779

Kellermann, K. I. 1964, $A$ p. J., 140, 969

Sharpless, S. 1959, Ap. J. Suppl, 4, 257.

\section{THE 8-14-MICRON APPEARANCE OF VENUS BEFORE THE 1964 CONJUNCTION*}

In an earlier paper (Murray, Wildey, and Westphal 1963) we have described the detailed mapping of the 8-14- $\mu$ radiation from Venus using the 200-inch Hale telescope. This paper extends that work over a period of about 7 months from December 15, 1963, to June 6,1964 . Detailed maps for 6 days are presented that show the morphology of the upper-atmosphere brightness temperature and illustrate a number of anomalous features near the cusps. The problem of atmospheric extinction and the derived brightness temperature is discussed.

The east-arm Cassegrainian focus of the Hale telescope was used again during twilight periods for these observations. The photometer-detector system was similar to that discussed by Westphal, Martz, and Murray (1963) but modified so that photographs could be taken of the image of the planet superimposed on the focal-plane diaphragm of the photometer. This procedure allowed more accurate positioning of the scan lines with respect to the visible image of the planet. Unfortunately, the infrared image, particularly at large zenith angles, was significantly displaced by differential refraction from the photographic image, requiring a shift of the data in the direction of altitude to produce coincidence. Figure 1 illustrates the appearance of the planet on May 8, 1964, with the focal-plane diaphragm superimposed. The admitting aperture was about $1^{\prime \prime}$ in diameter. The scanning proceeded from west to east along a constant declination by adjusting the hour-angle drive rate of the telescope at about $2500^{\prime \prime} / \mathrm{hr}$ slow. The scans were initiated near the north cusp and repeated each $2^{\prime \prime}-5^{\prime \prime}$ southward in declination. Declination scans were generally also made subsequently if time allowed. The anomalous areas near the southern cusp were generally studied by partial north to south scans at small increments of right ascension.

The photographs, taken during each scan, allowed a mutually coherent scan map to be made, locating each data point read from the strip-chart records.

The data were converted from deflections to numerical values from the strip charts on a spacing compatible with the angular resolution of the telescope-photometer system. These data were reduced to brightness temperatures, utilizing the method described by

\footnotetext{
* Contribution No. 1318 of the Division of Geological Sciences, California Institute of Technology, Pasadena, California.
} 
Murray and Wildey (1964), with an IBM 7094 computer. The temperatures were plotted on a base map constructed from the photographs as above and contoured to produce the brightness-temperature maps illustrated in Figure 2.

The deflections have been corrected for atmospheric extinction by a method that is to a certain extent arbitrary. On those nights when scans were made both in right ascension and declination, common points on the planet were measured with a significant time interval to allow the calculation of an extinction coefficient directly. Since the differential extinction correction is relatively insensitive to a choice of extinction model, we have chosen for convenience to use a "gray" model of the form

$$
I=I_{0} e^{-k m},
$$

where $I$ is observed flux intensity, $I_{0}$ is external flux intensity, $k$ is extinction coefficient, and $m$ is air mass. This procedure allows a quite accurate differential brightness-temperature map to be constructed.

The basic question of the absolute extraterrestrial brightness temperature for the subearth point of Venus observed in the 8-14- $\mu$ window has not been clarified by these data. Table 1 lists the extinction coefficients and derived peak-brightness temperatures computed from the data.

TABLE 1

MEASURED EXTinCtion COEFFicients AND DeRived PEAK BRightNess Temperatures of Venus

\begin{tabular}{l|c|c}
\hline \hline \multicolumn{1}{c|}{ Date } & $\begin{array}{c}\text { Extinction } \\
\text { Coefficient }\end{array}$ & $\begin{array}{c}\text { Peak Brightness } \\
\text { Temperature } \\
\left({ }^{\circ} \mathrm{K}\right)\end{array}$ \\
\hline December 15, 1963 & 0086 & 227 \\
April 14, 1964 & 193 & 206 \\
April 15, 1964 . & .102 & 205 \\
May 8, 1964. & 292 & 219 \\
May 26, 1964 & 076 & 214 \\
June 6, 1964... & 0129 & 217 \\
\hline
\end{tabular}

To correct the observed specific intensity properly one must know rather accurately the atmospheric extinction as a function of three variables: air mass, time, and wavelength. The 8-14- $\mu$ window is filled with absorption lines from water vapor, carbon dioxide, and ozone and has more or less "gray" absorption from atmospheric aerosols and the wings of the longer-wavelength water bands. As may be seen in Table 1, the net effect is quite variable and the choice of the form of the extinction controls the derived absolute temperatures. It seems clear that until extra-atmospheric fluxes in this wavelength region are known for some standard object, the question of absolute brightness temperature of Venus and possible variations in that quantity will be in doubt.

From the series of detailed maps it is clear that rather large, complicated changes are occurring in the part of the Cytherian atmosphere observed by $8-14-\mu$ emission. Several general features are apparent:

1. The extreme bilateral symmetry seen in December, 1962, was not present during these observations, although some such effect was still apparent. Whether this is related to observing the "opposite" side of the planet, i.e., the sunset side for a retrograde rotation in 1964, and the sunrise side in 1962, is not known. However, the variation of this effect is inconsistent with the hypothesis that the distribution of atmospheric boundary temperature depends only upon latitude.

2. The brightness temperature is higher at the antisolar point. This may be seen best on the maps for April, where the contours are most obviously displaced to the unillumi- 

diameter. 
nated side. That the maximum is not greatly displaced from the antisolar point may be seen on the June 6 map. Unfortunately, the absolute temperatures are of no help in resolving the details of this effect.

3. Small asymmetries and local anomalies are common on the maps. These irregularities are most common near the southern cusp. A detailed examination of the scans near that cusp indicate a real anomaly on every day of observation. These anomalies usually are small "plateaus" in the normal limb darkening similar to but usually less obvious than that shown in our earlier report (see Fig. 5 of Murray et al. 1963).
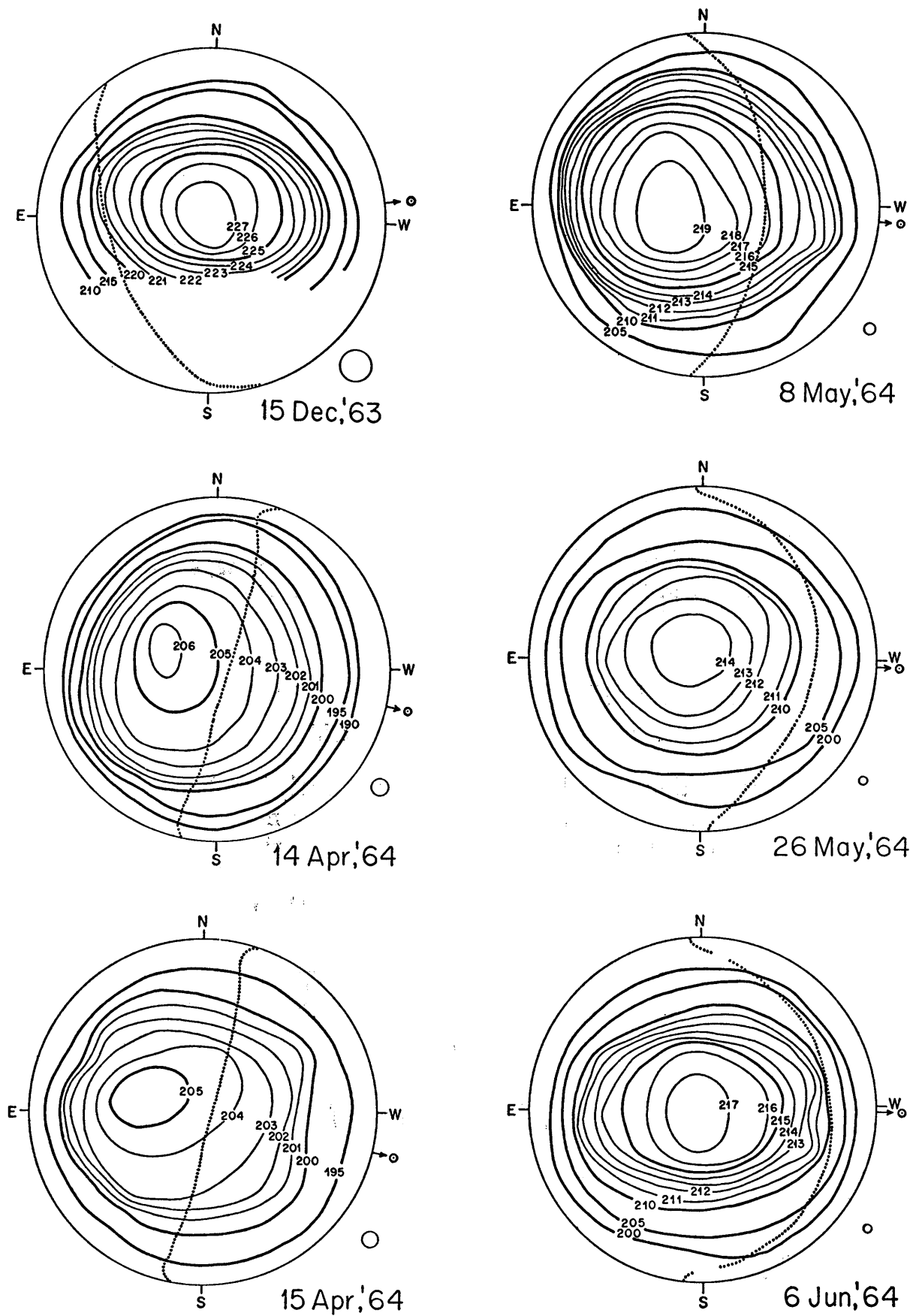

Fig. 2.-Brightness temperature maps of Venus. Direction to the Sun, measuring diaphragm size, and terminator are indicated. Brightness temperatures in degrees Kelvin. 


$$
\text { I': }
$$

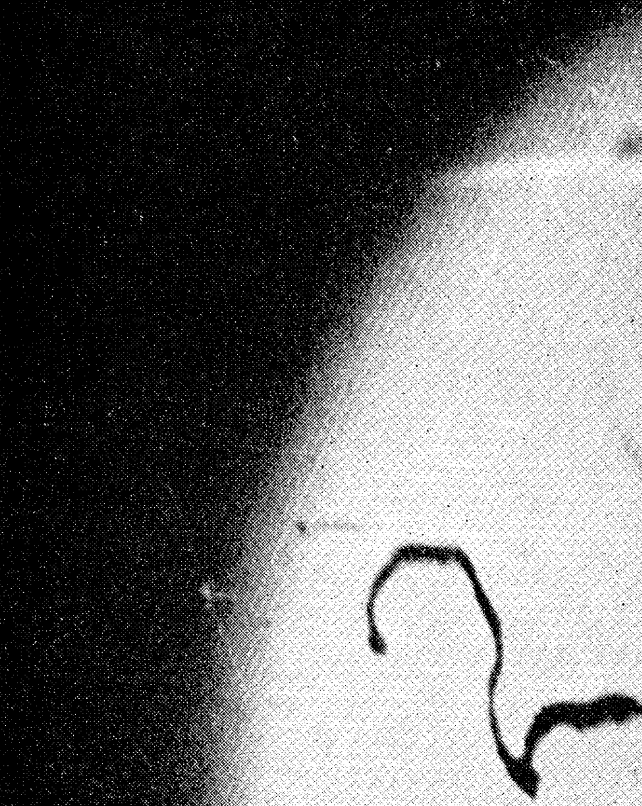

3. 1.-Photograph of Venus on May 8,1964, with the focal-plane diaphragm near the northern cusp. The planet is 3: meter. 